\title{
Preparation and characterisation of water-soluble phytosterol nanodispersions.
}

\begin{abstract}
The purpose of this study was to prepare and characterise water-soluble phytosterol nanodispersions for food formulation. The effects of several factors were examined: four different types of organic phases (hexane, isopropyl alcohol, ethanol and acetone), the organic to aqueous phase ratio and conventional homogenisation vs. high-pressure homogenisation. We demonstrated the feasibility of phytosterol nanodispersions production using an emulsification-evaporation technique. The results showed that hexane was able to produce the smallest particle size at a mean diameter of approximately $50 \mathrm{~nm}$ at monomodal distribution. Phytosterol nanodispersions prepared with a higher homogenisation pressure and a higher organic to aqueous phase ratio resulted in significantly larger phytosterol nanoparticles $(\mathrm{P}<0.05)$. Phytosterol loss after high-pressure homogenisation ranged from $3 \%$ to $28 \%$, and losses increased with increasing homogenisation pressure. Elimination of the organic phase by evaporation resulted in a phytosterol loss of $0.5-9 \%$.
\end{abstract}

Keyword: Phytosterol; Nanodispersions; Mean particle diameter; Primary homogenisation; High-pressure homogenisation. 